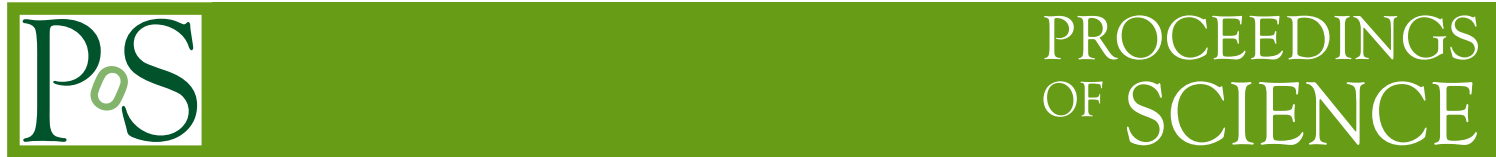

\title{
Neutron-rich Helium isotopes based on hyperspherical harmonics
}

\section{Sonia Bacca ${ }^{* t}$}

TRIUMF, 4004 Wesbrook Mall, Vancouver, BC V6T 2A3, Canada

E-mail: baccaetriumf.ca

\begin{abstract}
We present recent results for neutron-rich Helium isotopes obtained from the hyperspherical harmonics method. Ground-state properties, like the binding energy and the point-proton radius are shown for the two-neutron halo nucleus ${ }^{6} \mathrm{He}$ using two-body low-momentum interactions derived from chiral forces. The applicability of the method to the four-neutron halo nucleus ${ }^{8} \mathrm{He}$ is discussed. As an excited-state observable we present a recent calculation of the nuclear electric polarizability of ${ }^{6} \mathrm{He}$ from a semi-realistic potential. A comparison of the calculated quantities to experimental data is performed.
\end{abstract}

The 7th International Workshop on Chiral Dynamics, August 6 -10, 2012

Jefferson Lab, Newport News, Virginia, USA

\footnotetext{
* Speaker.

${ }^{\dagger}$ Thanks to my collaborators N. Barnea, R. Goerke and A. Schwenk for their help in obtaining these results.
} 


\section{Introduction}

The physics of light neutron-rich nuclei is particularly interesting, because of the appearance of their exotic structures, like those of halo nuclei. The lightest nuclei of this kind are found in the Helium isotope chain: ${ }^{6} \mathrm{He}$ as a two-neutron and ${ }^{8} \mathrm{He}$ as a four-neutron halo nucleus. They are both radioactive and undergo $\beta$-decay with a half life of $t_{1 / 2}=0.8 \mathrm{~s}$ and $0.1 \mathrm{~s}$, respectively. Despite the short life time, a combination of atomic and nuclear physics techniques, has enabled precise measurements of ground-state observables like the energy and the charge radius [1]. Excited-state properties, like electromagnetic transitions in the continuum have been investigated in the past with Coulomb dissociation experiments by Aumann et al. [2]. Tackling the theoretical study of these nuclei is very challenging, because one needs to simultaneously describe the small separation energy of the halo neutrons and the large radius of the whole system. Because they are light-mass nuclei, one can use ab-initio techniques to study them. Here, we will discuss the recent results obtained using the hyperspherical harmonics $(\mathrm{HH})$ method.

A major breakthrough in nuclear physics has been the development of chiral effective field theory, which is well routed to Quantum Chromo Dynamics. Even though several light nuclei have been investigated with chiral potentials, we are still missing a prediction of Helium halo nuclei from chiral Hamiltonians. Here, we show a first step taken in this direction by using low-momentum chiral two-nucleon forces.

The paper is organized as follows. In section 2 we will introduce the hyperspherical harmonics method. In section 3 and 4 we will present results for ground-state and excited-state properties, respectively. Finally, in section 5 we will draw some conclusions.

\section{Hyperspherical Harmonics}

Given the Hamiltonian $H$ we use the HH expansion to solve the Schrödinger equation. The HH method is typically a few-body method used for 3 and 4-body systems. Using the powerful antisymmetrization algorithm introduced in [3], it is possible to extend the method to a larger mass number and tackle Helium halo nuclei. The HH approach starts from the Jacobi coordinates

$$
\eta_{0}=\frac{1}{\sqrt{A}} \sum_{i=1}^{A} \mathbf{r}_{i}, \quad \eta_{k-1}=\sqrt{\frac{k-1}{k}}\left(\mathbf{r}_{k}-\frac{1}{k-1} \sum_{i=1}^{k-1} \mathbf{r}_{i}\right), k=2, \ldots, A,
$$

where $\mathbf{r}_{i}$ are the particle coordinates. Using the $\eta_{i}$ one can then transform to hyperspherical coor-

dinates composed of one hyperradial coordinate $\rho=\sqrt{\sum_{i=1}^{A-1} \eta_{i}^{2}}$ and a set of $(3 A-4)$ angles that we denote with $\Omega$ (for more details see [3]). Using this coordinates one can recursively construct the hyperspherical harmonics $\mathscr{Y}_{[K]}$ and use them as a complete basis to expand the wave function. Such expansion reads

$$
\Psi\left(\eta_{1}, \ldots, \eta_{A-1}, s_{1}, \ldots, s_{A}, t_{1}, \ldots, t_{A}\right)=\sum_{n}^{n_{\max }} \sum_{[K]}^{K_{\max }} C_{[K] n} R_{n}(\rho) \mathscr{Y}_{[K]}\left(\Omega, s_{1}, \ldots, s_{A}, t_{1}, \ldots, t_{A}\right),
$$

where $s_{i}$ and $t_{i}$ are the spin and isospin of the nucleon i, respectively; $C_{[K] n}$ is the coefficient of the expansion, labeled by $[K]$, which represents a cumulative quantum number that includes the 

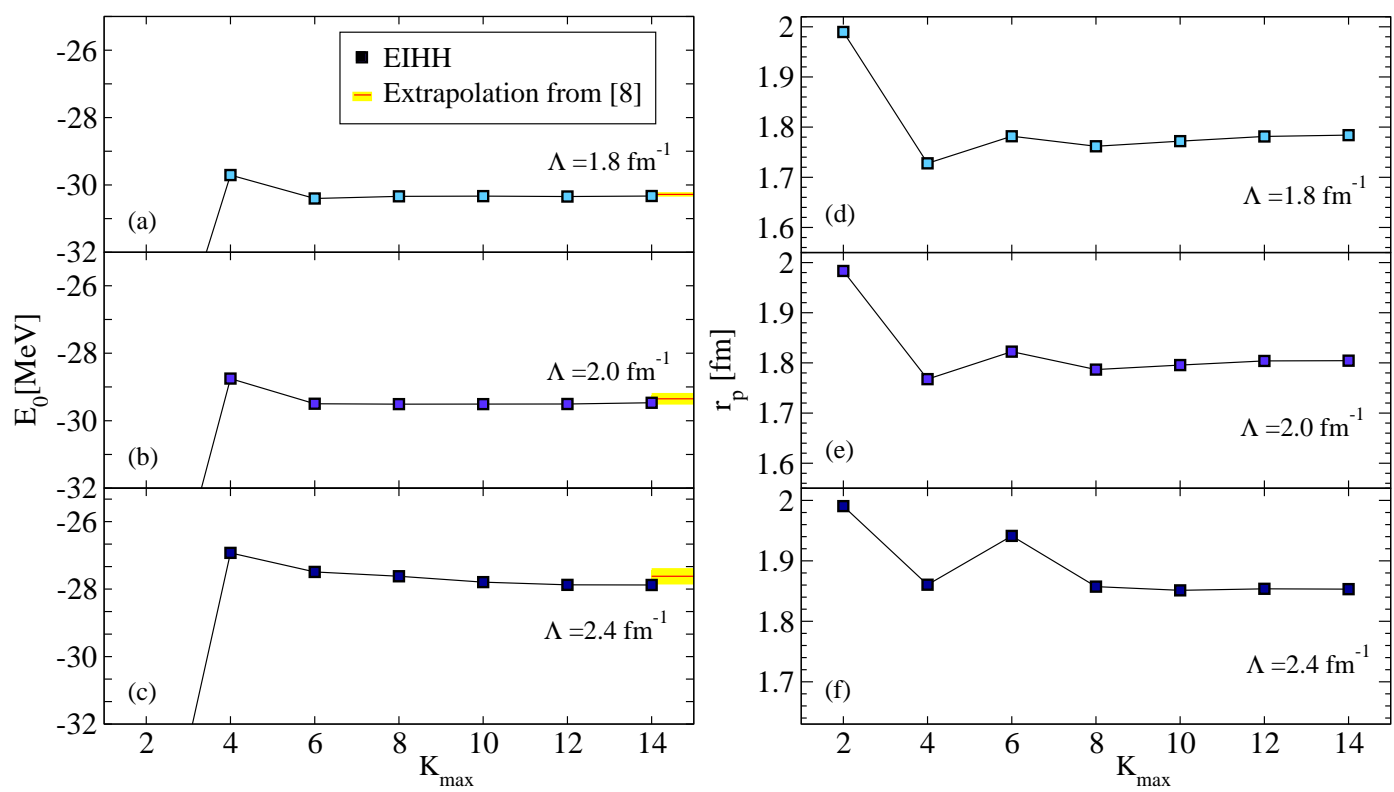

Figure 1: (Color online) The ${ }^{6} \mathrm{He}$ ground-state energy (left) and the point-proton radius (right) as functions of $K_{\max }$ obtained for three different values of the cutoff $\Lambda=1.8,2.0,2.4 \mathrm{fm}^{-1}$ of the $V_{\text {low } k}$ chiral potential.

grandangular momentum $K$; $n$ labels the hyperradial wave function $R_{n}(\rho)$. The latter is expanded in terms of the generalized Laguerre polynomials times an exponentially falling off function, which is essential to speed up the convergence of halo nuclei, due to their extended tail. To further increase the convergence rate of the calculations, we typically employ an effective interaction in the hyperspherical harmonics (EIHH), as first introduced in [4].

\section{Results for ground state properties}

In the following, we present our results for the ground state energy and the point-proton radius calculated using the hyperspherical harmonics method. As input Hamiltonian we employ a class of low-momentum potentials, which are obtained applying a $V_{\text {low } k}$ procedure [5] on a starting chiral two-body potential [6] at next-to-next-to-next-to leading order $\left(\mathrm{N}^{3} \mathrm{LO}\right)$.

In Fig. 1, we show the ${ }^{6} \mathrm{He}$ convergence patterns of the EIHH method for the ground-state energy and the point-proton radius as a function of the maximal grandangular momentum [7]. Three different cutoffs $\Lambda=1.8,2.0,2.4 \mathrm{fm}^{-1}$ of the $V_{\text {low } k}$ chiral potential are shown. One observes that the convergence is very nice for all $\Lambda^{\prime} s$, even for the largest cutoff. Concerning the energy we also show that the extrapolated results from previous work on hyperspherical harmonics [8] agree nicely with EIHH. The convergence rate is very good also for the point-proton radius, which allows us to provide solid results for ${ }^{6} \mathrm{He}$.

We have also explored the four-neutron halo nucleus ${ }^{8} \mathrm{He}$ with hyperspherical harmonics. In Fig. 2, we show the ${ }^{8} \mathrm{He}$ ground state energy from a $V_{\text {low } k}$ chiral potential with $\Lambda=1.8 \mathrm{fm}^{-1}$ as a function of $K_{\max }$. We present both the variational HH expansion (where we do not apply the effective interaction) and the EIHH results. The convergence for ${ }^{8} \mathrm{He}$ is quite slow, as indicated by the fact that the $\mathrm{HH}$ and $\mathrm{EIHH}$ patterns do not merge yet at $K_{\max }=10$. Interestingly, an ex- 


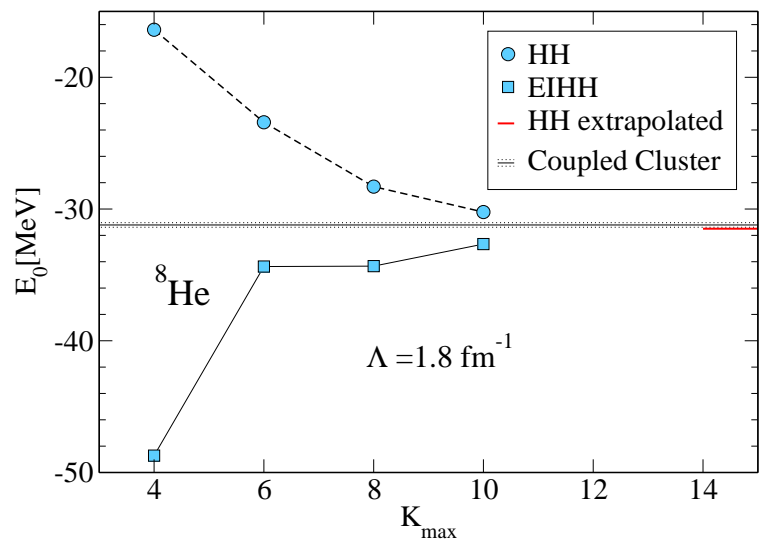

Figure 2: (Color online) The ${ }^{8} \mathrm{He}$ ground-state energy calculated with the $\mathrm{HH}$ and EIHH methods as a function of $K_{\max }$ obtained for a cutoff of $\Lambda=1.8 \mathrm{fm}^{-1}$ of the $V_{\text {low } k}$ chiral potential. The extrapolated HH results are shown as a reference and compared to the Coupled-Cluster results.

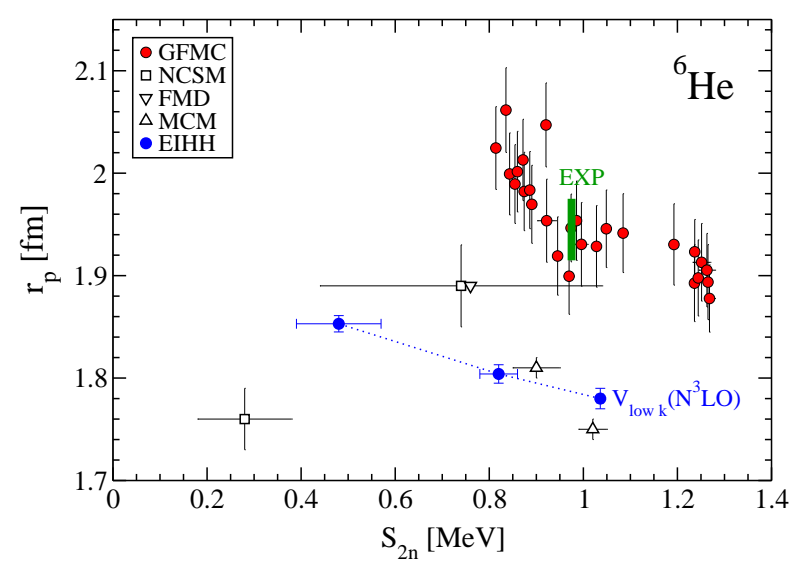

Figure 3: (Color online) Correlation plot of the ${ }^{6} \mathrm{He}$ point-proton radius versus the two-neutron separation energy $S_{2 n}$. The experimental range is compared to theory based on different ab-initio methods (see text).

trapolation of the variational $\mathrm{HH}$ data lies close to the Coupled-Cluster result from [8] with the same interaction. Nevertheless, at the moment we are not able to provide precise results for ${ }^{8} \mathrm{He}$ from hyperspherical harmonics. Thus, we concentrate on analyzing the correlation between energy and radius just for ${ }^{6} \mathrm{He}$. In Fig. 3, we plot $r_{\mathrm{p}}$ versus the two-neutron separation energy $S_{2 n}$ and present a combined comparison of our results to experiment and other ab-initio calculations: Green's Function Monte Carlo (GFMC), No Core Shell Model (NCSM), Fermionic Molecular Dynamics (FMD) and Microscopic Cluster Model (MCM) (see also [7] and references therein). The cutoff dependence of our results with $V_{\text {low } k}$ allows us to study the correlation between these observables: the radius increases as the separation energy decreases. Our calculations do not reproduce simultaneously $r_{\mathrm{p}}$ and $S_{2 n}$ : there exists an optimal value of $\Lambda$ where $S_{2 n}$ is predicted in accordance with experiment, but $r_{\mathrm{p}}$ is not reproduced and vice-versa. Also, we would like to note that other calculations which omit $3 N F$, (all except from the GFMC) do not go though the experimental band. 

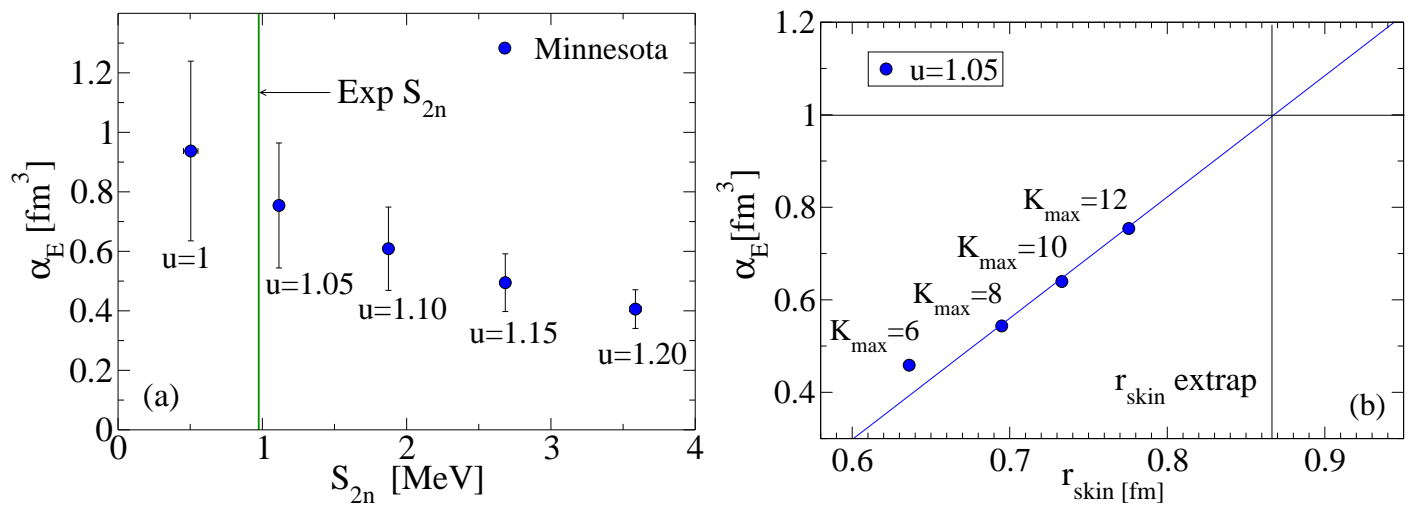

Figure 4: (Color online) Panel (a): The correlation between $\alpha_{E}$ and $S_{2 n}$ in ${ }^{6} \mathrm{He}$ obtained with the the Minnesota potential varying the parameter $u$. Panel (b): The correlation between $r_{\text {skin }}$ and $\alpha_{E}$ in different model spaces (different $K_{\max }$ ). Shown is also the extrapolated value of $r_{\text {skin }}$, which is used to estimate $\alpha_{E}$.

This points towards the importance of including three-nucleon forces in the Hamiltonian.

\section{Results for excited state properties}

As an example of excited-state properties of halo nuclei we report about our recent calculation of the electric dipole polarizability $\alpha_{E}$ of ${ }^{6} \mathrm{He}$ [9]. $\alpha_{E}$ is related to the inelastic response of the nucleus to an externally applied electric field and is relevant in the extraction of nuclear quantities from atomic spectroscopic measurements. The atomic energy levels, in fact, are affected by polarization of the nucleus due to the electric field of the surrounding electrons. The polarizability of ${ }^{6} \mathrm{He}$ could be extracted from Coulomb dissociation measurement of the dipole transition by Aumann et al. [2] and was reported in Ref. [10] to be much bigger than the polarizability of ${ }^{4} \mathrm{He}$. The electric dipole polarizability is defined by

$$
\alpha_{E}=2 \alpha \sum_{f \neq 0} \frac{\left|\left\langle\Psi_{f}|E 1| \Psi_{0}\right\rangle\right|^{2}}{E_{f}-E_{0}},
$$

where $\left|\Psi_{0 / f}\right\rangle$ is the ground state and final state of the nucleus and $E 1$ is the dipole operator. Because it requires the knowledge of the dipole spectrum of the nucleus, its theoretical evaluation is more involved than a bound-state calculation. We perform our calculation with the EIHH method by using the Lanczos algorithm with a starting dipole pivot as explained in [9]. As nuclear potential we chose the simple semi-realistic Minnesota force, which reproduces the experimental value of the polarizability of ${ }^{4} \mathrm{He}$ reasonably well. Within this force model we can add attractive $P$-wave interactions by changing the parameter $u$ (see [9] for details). This mostly affects ${ }^{6} \mathrm{He}$, without substantially changing ${ }^{4} \mathrm{He}$. By varying $u$ we first observe a correlation of $\alpha_{E}$ vs $S_{2 n}$, as shown in Fig. 4(a). We have chosen $u$ so that the halo feature, represented by $S_{2 n}$, is reproduced. We then study the correlations between $\alpha_{E}$ and the skin radius $r_{\text {skin }}=r_{n}-r_{p}$, where $r_{n}$ is the mean pointneutron radius. By varying the model space we observed that $\alpha_{E}$ and $r_{\text {skin }}$ are correlated linearly for $K_{\max } \geq 6$ as $\alpha_{E}=a+b r_{\text {skin. }}$. From our theoretical data we fit the coefficients $a$ and $b$ and then we used them to estimate the polarizability out of a bound-state calculation of the skin radius. The 
calculation of $r_{\text {skin }}$, in fact, does not require an expansion on the dipole excited states and as such is less computationally demanding and can be performed for larger model spaces $\left(K_{\max }=16\right)$ and then extrapolated exponentially, leading to $r_{\text {skin }}=0.87(5) \mathrm{fm}$. Using our extrapolated skin radius and the linear dependence, we estimate the theoretical nuclear electric polarizability of ${ }^{6} \mathrm{He}$ to be $\alpha_{E}=1.00(14) \mathrm{fm}^{3}$. The error bar is obtained by propagating the errors on $a, b$ and $r_{\text {skin. }}$. We observe that our theoretical estimate is about a factor of two smaller than the experimental value of $\alpha_{E}^{\exp }=1.99(40) \mathrm{fm}^{3}$ [10]. This points toward a potential disagreement between theory and experiment. Investigations with chiral potentials can possibly help understanding this discrepancy.

\section{Conclusions}

In conclusion, we have presented our recent results on Helium halo nuclei from hyperspherical harmonics. The binding energy and the radius can be precisely calculated for ${ }^{6} \mathrm{He}$ using chiral low-momentum two-body forces. The obtained cutoff dependence together with a comparison to the experiment serves to highlight the importance of three-nucleon forces. We also discussed the nuclear dipole polarizability of ${ }^{6} \mathrm{He}$ as an excited state observable, which has recently attracted attention. Our estimate from simplified nuclear potentials leads to a disagreement with experimental data, which will be hopefully clarified in the future when realistic chiral potentials will be used.

\section{References}

[1] M. Brodeur et al., First direct mass measurement of the two-neutron halo nucleus ${ }^{6} \mathrm{He}$ and improved mass for the four-neutron halo ${ }^{8} \mathrm{He}$, Phys. Rev. Lett. 108, 052504 (2012) [arXiv: 1107.1684 ].

[2] T. Aumann et al., Continuum excitations in ${ }^{6} H e$, Phys. Rev. C 59, 1252 (1999).

[3] N. Barnea and A. Novoselsky, Hyperspherical wave functions with orthogonal and permutational symmetry, Phys. Rev. A 57, 48 (1998).

[4] N. Barnea et al., State dependent effective interaction for the hyperspherical formalism, Phys. Rev. C 61, 054001 (2000) [nucl-th/9910062].

[5] S. K. Bogner et al., From low-momentum interactions to nuclear structure, Prog. Part. Nucl. Phys. 65, 94 (2010) [arXiv: 0912.3688].

[6] D. R. Entem and R. Machleidt, Accurate charge-dependent nucleon-nucleon potential at fourth order of chiral perturbation theory, Phys. Rev. C 68, 041001(R) (2003) [nucl-th/ 0304018 ].

[7] S. Bacca et al., Matter and charge radius of ${ }^{6} \mathrm{He}$ in the hyperspherical harmonics approach, Phys. Rev. C 86, 034321 (2012) [arXiv: 1202 . 0516].

[8] S. Bacca et al., Helium halo nuclei from low-momentum interactions, Eur. Phys. J. A 42, 553 (2009) [arXiv:0902.1696].

[9] R. Goerke et al., Nuclear electric polarizability of ${ }^{6} H e$, Phys. Rev. C 86, 064316 (2012) [arXiv:1209.2468].

[10] K. Pachucki, A.M. Moro, Nuclear polarizability of helium isotopes in atomic transitions, Phys. Rev. A 75, 032521 (2007) [nucl-th/ 0612065 ]. 